Research Paper

\title{
Combination of Heel-strike like Mechanical Loading with Deproteinized Cancellous Bone Scaffold Implantation to Repair Segmental Bone Defects in Rabbits
}

Guofeng Huang*, Guojun Liu*, Feng Zhang*, Jianting Gao, Jiangze Wang, Qi Chen, Benwen Wu, Zhenqi Ding ${ }^{凶}$, Taoyi Cai ${ }^{\bowtie}$

Center for Orthopedics, Affiliated Southeast Hospital of Xiamen University/175th Hospital of People's Liberation Army, Zhangzhou, P. R. China, 363000

* Co-first authors: Guofeng Huang, Guojun Liu, and Feng Zhang contributed equally to this article.

$\triangle$ Corresponding author: Zhenqi Ding, Department of Orthopedic Surgery, Affiliated Southeast Hospital of Xiamen University/175th Hospital of People's Liberation Army, Zhangzhou, P. R. China, TEL: +86-0596-2931538, E-mail: dzqi@xmu.edu.cn and Taoyi Cai, Department of Orthopedic Surgery, Affiliated Southeast Hospital of Xiamen University/175th Hospital of People's Liberation Army, Zhangzhou, P. R. China, TEL: +86-0596-2931538, E-mail: ctyi175@163.com

(C) Ivyspring International Publisher. This is an open access article distributed under the terms of the Creative Commons Attribution (CC BY-NC) license (https://creativecommons.org/licenses/by-nc/4.0/). See http://ivyspring.com/terms for full terms and conditions.

Received: 2017.02.11; Accepted: 2017.05.17; Published: 2017.07.19

\begin{abstract}
Under physiological conditions bone defects often occur at mechanical load bearing sites and bone substitutes used for regeneration should be similarly subjected to mechanical loading stress. In this study, we investigated whether a novel heel-strike like mechanical loading method can be used as a complementary therapy to promote bone regeneration following bone substitute grafting. To test this, three groups of rabbits with segmental bone defects in the tibia were implanted with bovine deproteinized cancellous bone scaffold (DCBS), with one group also receiving heel-strike like mechanical loading generated by a rap stress stimulator. From weeks 4-12 post-operation $\mathrm{X}$-ray and micro-CT scanning showed that rabbits receiving combination therapy had significantly more callus at the bone defect. Moreover, bone defects in the combination group were completely replaced with new bone at week 12 , while the DCBS implantation alone group healed only partially and rabbits receiving neither DCBS nor mechanical loading developed only small calluses throughout the observation period. Analysis of micro-CT scanning results demonstrated that new bone density in the combination group was significantly higher than the DCBS only group at weeks 4 and $12(p<0.05)$. H\&E staining results also indicated a significantly higher percentage of new bone in the bone defect area and a lower percentage of residual scaffold in the combination group compared to the DCBS only group $(p<0.05)$. Thus, this heel-strike like mechanical loading method appears to accelerate bone regeneration following substitute implantation by restoring a local mechanical loading environment in segmental bone defects.
\end{abstract}

Key words: segmental bone defect, heel-strike like mechanical loading, deproteinized cancellous bone scaffold, bone substitutes, bone regeneration

\section{Introduction}

The treatment of segmental bone defects caused by trauma, infection, or bone tumor resection remains a great challenge for orthopedic surgeons, and the growing clinical demands for bone substitutes highlights the need for researchers to both develop new bone scaffolds and improve the process of bone regeneration [1]. The latter has been the focus of many studies and several tissue-engineering techniques have been proposed to enhance osteogenesis inside bone scaffolds [2], such as pre-seeding scaffolds with mesenchymal stem cells [3], delivering growth factors like bone morphogenetic protein 2 (BMP-2) or 
transforming growth factor $\beta 1$ (TGF $\beta-1$ ) within scaffolds [4-5], or pre-vascularization of scaffolds in vitro before implantation [6]. While each of these methods have been successful to some extent they all have disadvantages, including extended preparation time and a high cost for the final product.

Mechanical loading is a potential alternative for enhancing bone regeneration that address both of these concerns. The regulatory effect of mechanical loading on bone cells and normal bone architecture, as well as its critical role in the bone healing process, are widely acknowledged [7]. However, in segmental bone defect situations high-energy trauma or iatrogenic internal/external fixations cause damage to the mechanical loading environment, resulting in mechanical loading deficiency. Several methods have been proposed to restore the mechanical loading environment: in vitro studies have shown fluid flow enhances expression of the osteoblastic phenotype in mesenchymal stromal cells and improves mineralization [8], while some in vivo mechanical loading methods have also been proven to improve bone formation inside scaffolds [9-10]. However, the in vitro conditions used are substantially different in their environmental conditions and in the manner of new-bone formation than what occurs in vivo, while most of the in vivo experiments employed invasive mechanical loading methods. More importantly, the bone defect models used were not consistent with clinical situations because they did not take internal fixation factors into consideration.

To restore the mechanical environment in a bone fracture model we have invented a novel method of mechanical loading. We previously demonstrated that rapping the heels of lower limbs with a hammer can generate a mechanical loading stress conducted through the longitudinal axis of the lower limb that produces pressure on the fracture end, mimicking the effects of walking [11]. In the present study, we tested whether combining this heel-strike like mechanical loading with deproteinized cancellous bone scaffold (DCBS) implantation to repair segmental bone defects would enhance bone regeneration. We implanted bone scaffolds fixed with plates and screws in rabbits with segmental tibial bone defects, treating one group with mechanical loading stress as well, and tracked the progress of bone repair by X-ray, micro-computed tomography (CT), and histological analysis. Longitudinal analysis was employed to investigate the effect of loading on bone formation throughout the healing process. Our results show that this novel heel-strike like mechanical loading method can repair local mechanical loading deficiency in segmental bone defects and thus enhance bone regeneration after bone scaffold grafting.

\section{Materials and Methods}

\section{Preparation of deproteinized cancellous bone scaffold}

The distal femurs of 6-month-old cattle were collected and the cartilage and cortical bones were eliminated, leaving only the cancellous bone which was cut into $1.5 \mathrm{~cm}$ long cylinders (diameter $=1 \mathrm{~cm}$ ). A deproteinized cancellous bone scaffold (DCBS) was prepared as previously described [12]. Briefly, bones were immersed in $20 \%$ hydrogen peroxide, deproteinized for $72 \mathrm{~h}$, incubated at $37^{\circ} \mathrm{C}$ and periodically degreased with diethyl ether for $48 \mathrm{~h}$, and then freeze-dried. The DCBSs were disinfected with ethylene oxide and sealed for later use. Scanning electron microscopy (SEM) was used to observe and analyze the surface microstructure. Mechanical compression tests were performed on the six scaffolds using an Instron 5567 (Instron Corp, Norwood, MA) in displacement-control mode at a rate of $1.2 \mu \mathrm{m} / \mathrm{s}$ and a corresponding strain rate of $6 \times 10^{-4} / \mathrm{s}$. Load and displacement data were used to determine the maximum compressive strength for each sample.

\section{Animal segmental bone defect model}

All experiments were performed in accordance with NIH principles of laboratory animal care $(\mathrm{NIH}$ publication No. 85-23, revised 1985) and ethics approval was obtained from the institutional Review Board or Animal Care and Use Committee of our hospital. Sixty 6-month-old New Zealand rabbits were randomly divided into three groups: Group A (n $=30), B(n=30)$ and $C(n=20)$. Segmental bone defects measuring $5 \mathrm{~mm}$ were created in the right tibias and the fracture ends were fixed using an assisted steel plate (Fig. 1A) as previously described [13]. All animals were deprived of food and water for $6 \mathrm{~h}$ before the surgery. Briefly, animals were anesthetized by intravenous ketamine and the surgical site was disinfected before a $7 \mathrm{~cm}$ long vertical skin incision was made at the anterior-medial of the right tibia to separate the subcutaneous tissue and deep fascia, exposing the tibia. A $5 \mathrm{~mm}$ long tibial bone section and its periosteum were removed using a fine electric saw and the fracture ends were fixed with one piece of 10-hole common steel plate, which was secured opposite the fibula with six tapping screws (China Suzhou Kangli Orthopedics Instrument Co., Ltd.). All rabbits were given a subcutaneous injection of penicillin $(400,000 \mathrm{U} / \mathrm{d})$ to prevent infection and analgesia treatment for $3 \mathrm{~d}$. All incisions were completely healed with no sign of infection at 1 week post-operation. The wounded leg was immobilized using a tubular plaster to prevent the rabbits from walking and reduce interference with the 

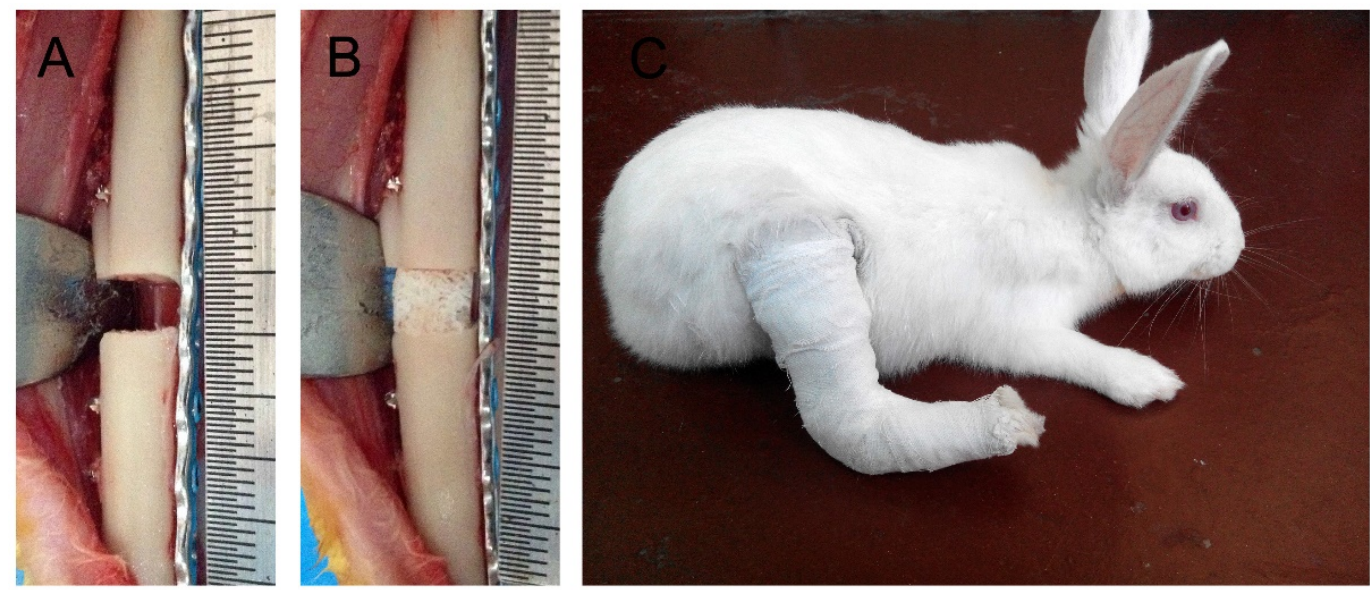

Figure 1. Surgical procedure for creation of rabbit tibia segmental bone defects. A: A 5-mm tibia section was removed and the fracture ends were fixed tightly with steel plates and screws. B: A suitable DCBS was grafted into the bone defect area. C: The wounded leg was immobilized with tubular plaster to prevent the rabbits from walking.
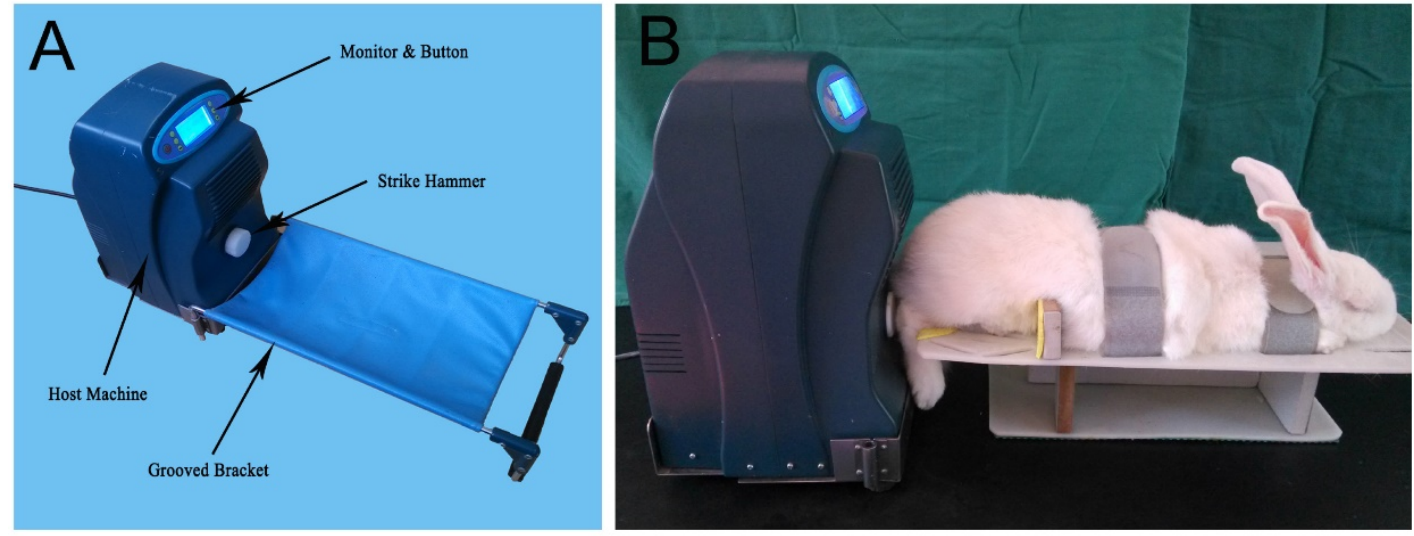

Figure 2. Independently designed in vitro rap stress stimulator. A: Appearance and components. B: Application of in vitro rap stress stimulator to anesthetized rabbits to generate mechanical loading stimulation.

mechanical loading treatment (Fig. 1C) [13].

\section{DCBS grafting and heel-strike like mechanical stimulus}

Suitable DCBSs were grafted into the bone defects of Groups A and B during surgery (Fig. 1B) and rabbits in Group A also received heel-strike like mechanical loading using an in vitro rap stress stimulator applied to the end of the tibia. The rap stress stimulator is composed of the host machine and a connected groove bracket (Fig. 2A) and contains a stepping motor, an integrated circuit plate, and a strike hammer. Rabbits were anesthetized with isopentane and fixed on the rapping platform (Fig. 2B) with the right tibia set horizontally on the fixer and the heel aligned with the hammer center. Stress generated by rapping the heel with the strike hammer is conducted through the longitudinal axis of the lower limb, simulating the pressure on the lower limb fracture that would be induced by walking [11]. The rabbits received this heel-strike like mechanical loading stimulus twice a day for $30 \mathrm{~min}$ per session, beginning eight days after the operation and lasting for 12 weeks. As in our previous study the mechanical rap parameters were set as $15 \mathrm{~N}$ (intensity), $1 \mathrm{~Hz}$ (frequency), $5 \mathrm{~s}$ (per time), and $3 \mathrm{~s}$ (time interval) [11]. After each rapping session the leg was fixed again with plaster. Group B rabbits were grafted with suitable DCBSs but not treated with mechanical loading and Group C served as an untreated control.

\section{Observation of bone regeneration}

Follow-up examinations were performed at 2, 4, 8 , and 12 weeks post-operation. Bone regeneration was tracked continuously by X-ray in 5 individuals from each group for all 12 weeks. For visual observation, micro-CT, and H\&E analysis samples were obtained from 5 rabbits from each group sacrificed at each time point.

X-ray scanning and scoring: Anterior-posterior $\mathrm{X}$-ray scans of the right tibia were taken and the Lane-Sandhu X-ray scoring standard was used for quantitative analysis of callus and reconstruction conditions $(n=5)$. 

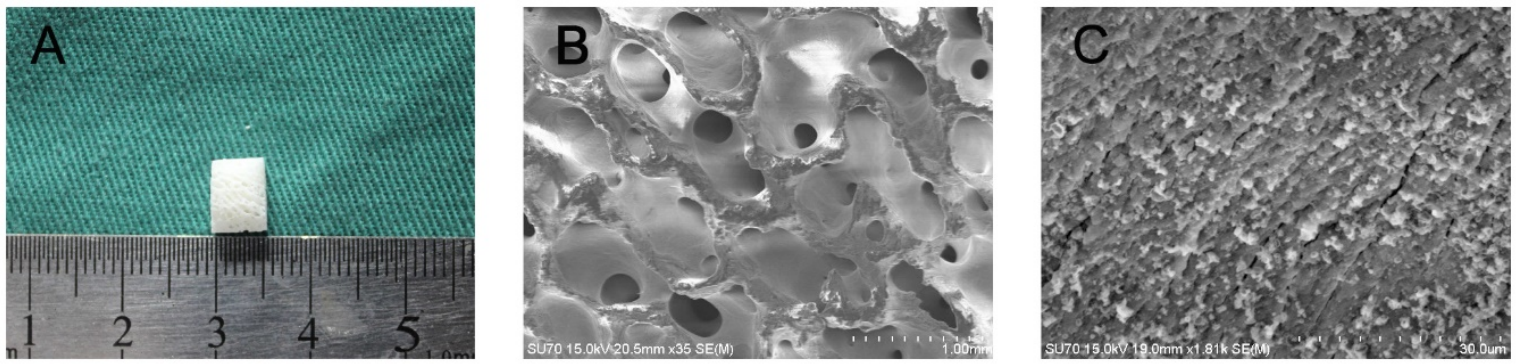

Figure 3. SEM analysis of DCBS. A: Gross appearance of DCBS. B: 3D structure showing uniform pore structures (6000×). C: Uniform particulate surface structure of DCBS $(7000 \times)$.

Visual inspection: Samples were collected from rabbits sacrificed by hyperanesthesia and were visually inspected for the degree of bone defect repair and presence of residual DCBS scaffold $(n=5)$.

Micro-CT scanning and quantitative analysis: Samples from both the normal and tibial bone defect ends were scanned with high-resolution $(12 \mu \mathrm{m})$ micro-CT and a 3D image was reconstructed. The area of the DCBS scaffold and the regenerated bone volume and density were analyzed using Image-Pro Plus Software (Media Cybernetics, Rockville, MD, USA) $(n=5)$.

H\&E staining and quantitative analysis: $3 \mathrm{~mm}$ bone tissue samples were collected from both ends of the defects, fixed in $10 \%$ triformol for $48 \mathrm{~h}$, dehydrated, and embedded in paraffin. Undercalcificated bone slicing was used for sagittal viewing. H\&E staining was performed after conventional slicing to observe bone regeneration and DCBS degradation at the sites of bone defects was observed under a light microscope. Images were collected and blindly analyzed with Image-Pro Plus by an independent technician to calculate the percentage of regenerated bones and residual DCBS scaffold in the total bone defect area.

Biomechanical analysis: To evaluate functional bone regeneration after 12 weeks we performed a three-point bending test $(n=5)$ using a universal testing machine (DE-WI-IS-001; Xiamen Double Engine Medical Material CO., LTD, China). The maximum load $(\mathrm{N})$ and stiffness $(\mathrm{N} / \mathrm{mm})$ were interpreted and calculated from the load-deflection curve. Six normal left tibiae were used as controls.

\section{Statistical analysis}

SPSS 19.0 was used for all data analysis. Normal distribution and error uniformity were evaluated with the Shapiro-Wilk and Levene method and are expressed as $x \pm s$. One-way ANOVA was used for comparison between the three groups at each testing time. If the difference was statistically significant, LSD-t was used for pairwise comparison. Measurements for Groups A and B at the same testing time points were compared through independent sample t-tests. $P<0.05$ indicates statistical significance.

\section{Results}

\section{Characteristics of DCBS scaffold}

SEM observation of DCBS confirmed uniform porosity $(60 \% \pm 12 \%)$ with an average pore diameter between 100-500 $\mu \mathrm{m}$. DCBS also had a rough particulate surface structure that benefits cell adhesion and crawling (Fig. 3) and a maximum compressive strength of $72.67 \pm 18.94 \mathrm{MPa}$.

\section{$X$-ray examination of bone defect repair process}

All rabbits were subjected to X-ray examination at 2, 4, 8, and 12 weeks post-operation. After 2 weeks neither Group A nor B had absorbed the DCBS but, both showed callus formation at the bone defect sites, though callus size in Group B was significantly smaller (Fig. 4A, B). In contrast, almost no callus was observed in Group C (Fig. 4C). In Group A the fibula side calluses increased substantially by week 4 and some of the DCBS was substituted by regenerated bones, but while Group B callus formation also increased greatly, the increase was smaller and there was no absorption of DCBS. Untreated animals in Group C only formed small calluses at the bone defect area. At 8 weeks post-operation there was substantial new bone formation in Group A, especially on the area opposite the steel plate side, and new bone connected the two fracture ends. Bone remodeling was also observed and the majority of the DCBS had been absorbed. Although the calluses in Group B also increased significantly bone remodeling was not prominent and most of the DCBS remained. Callus formation, bone remodeling, recanalization of the medullary cavity and degradation of the DCBS were complete in Group A after 12 weeks, but although we observed a further increase in Group B calluses with a calculated density similar to that of cortical bone some DCBS remained visible at the steel plate side. However, in Group C only a very few bridging 
calluses formed at the fibula side and the bone defect remained unrepaired even at week 12. Subsequent analysis with Lane-Sandhu X-ray scoring (Fig. 4D) resulted in a significantly higher score in Group A than Groups B and C at 4 weeks post-operation, with Group B as well as Group A scoring significantly higher than Group C $(p<0.05)$.
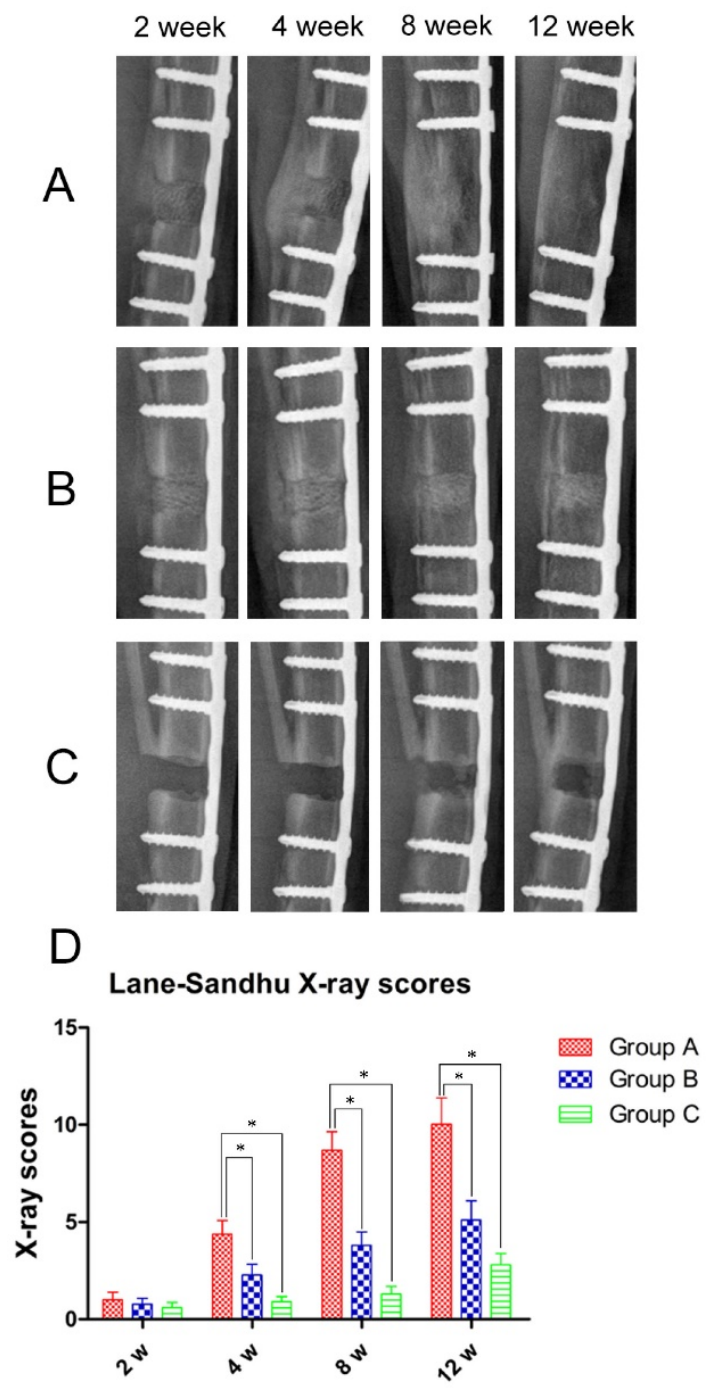

Figure 4. X-ray examination and Lane-Sandhu scoring. $X$-ray images of $A$ : Group A, B: Group B, and C: Group C at 2, 4, 8, and 12 weeks post-operation. $D$ : Lane-Sandhu scoring indicating significantly higher scores in callus and bone regeneration in Group A compared to Groups B and C beginning 4 weeks post-operation. $n=5, * p<0.05$.

\section{Gross observation of bone defect repair}

To confirm our X-ray analysis findings we collected bone specimens at 12 weeks post-operation for gross observation of bone repair. In Group A the DCBS was fully degraded and the bone defect was completely filled with regenerated bone that had been remodeled into cortical bone, indicating a perfect bone repair effect (Fig. 5A). Although we observed profuse bone regeneration and remodeling in Group
$B$ as well, residual DCBS still remained, especially at the area of the steel plate (Fig. 5B). In Group C there was still only a little callus formation on the side opposite the steel plate and the bone defect was filled with scars and granulated tissues (Fig. 5C). Notably, we found no evidence of bone fracture or internal fixation failure caused by rap mechanical stimulation throughout the experiment, indicating the safety of this treatment approach. However, two rabbits in the control group walked with wounded legs after biting their plaster, loosening the screws and requiring a second internal fixation.
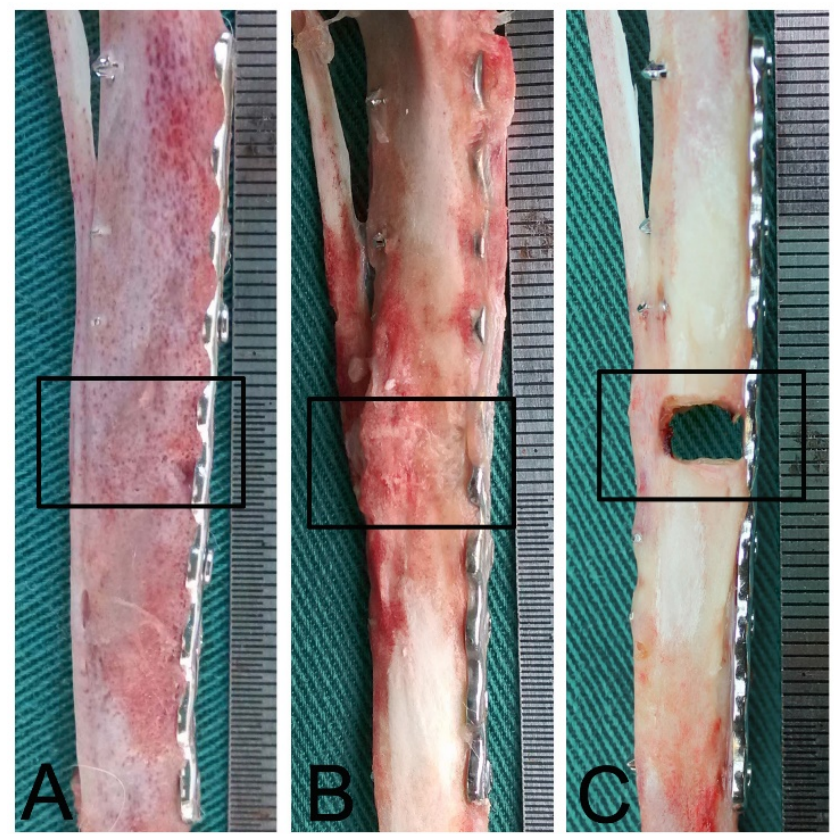

Figure 5. Gross observations 12 weeks after bone substitute grafting. A: The bone defect was completely repaired and the DCBS degraded in Group A, which received DCBS and mechanical loading stimulation group. B: In Group B, which only received DCBS grafting, the bone defect areas are partially repaired but some scaffold remains. C: The control group, Group C, received neither DCBS nor mechanical loading and shows only a small degree of callus formation. $\mathrm{n}=5$.

\section{Micro-CT scanning and analysis of regenerated bone}

To further quantify bone tissue regeneration we used micro-CT scanning. In keeping with our visual observations, at 4 weeks post-operation animals in Group A developed profuse callus growth, mainly at the end opposite of the steel plate, but most of the DCBS at the steel plate was retained. However, after 12 weeks the bone defect was completely repaired and the DCBS was replaced by regenerated bones, indicating good reconstruction (Fig. 6A). Similarly, Group B animals also produced new callus at the opposite side of the steel plate at 4 weeks post-operation but retained most of the DCBS. Although most bone defect areas were filled with 
4 week
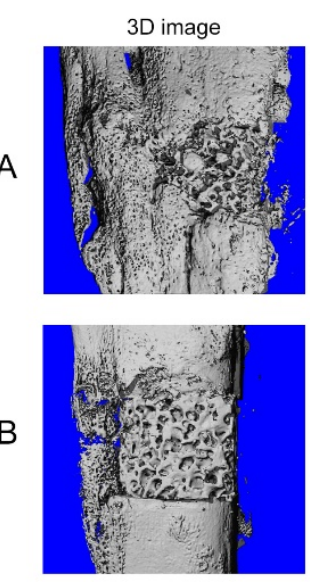
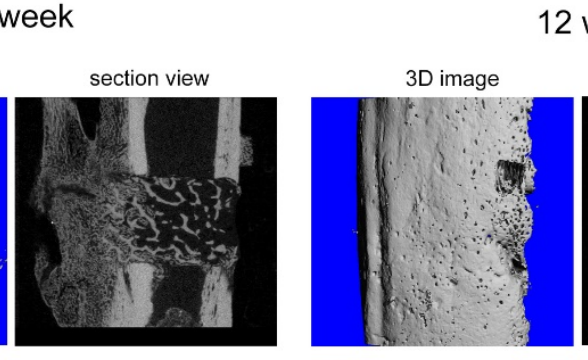

12 week
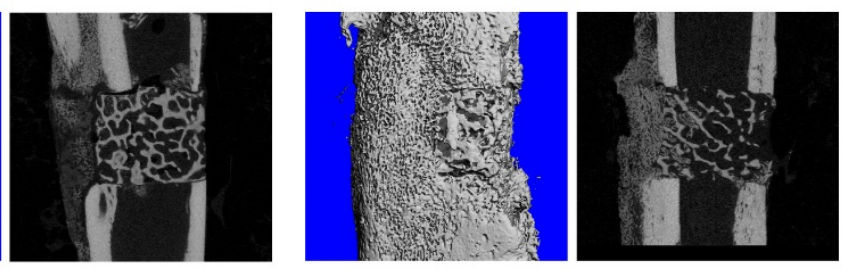

C
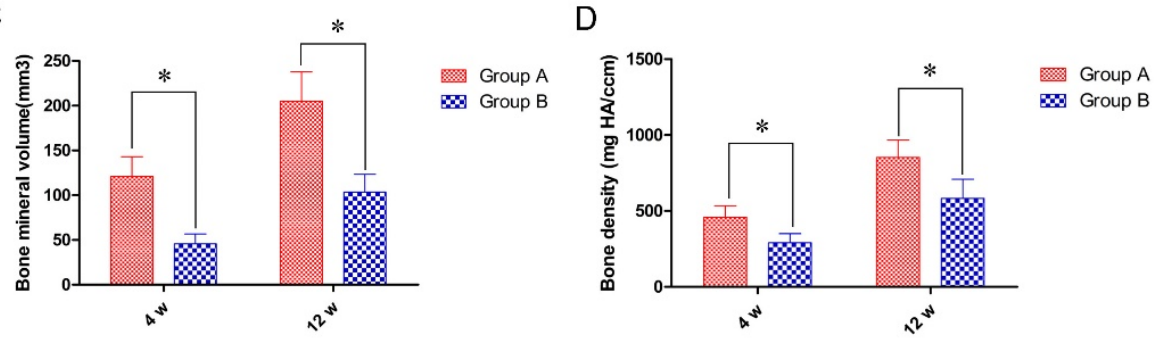

Figure 6. Micro-CT scanning results and determination of regenerated bone volumes and densities. Micro-CT images of A: Group A and B: Group B bones at 4 and 12 weeks post-operation. At both time points Group A had significantly higher regenerated bone C: volumes and D: densities than $G r o u p ~ B$. $n=5$, * $p<0.05$.

regenerated bones at 12 weeks post-operation the reconstructed callus was poorer than in Group A and we still detected residual DCBS (Fig. 6B). Using the micro-CT scanning data we were also able to determine the regenerated bone densities: in Group A the average densities were $458.2 \pm 76.3 \mathrm{mg} \mathrm{HA} / \mathrm{ccm}$ at 4 weeks and $852.8 \pm 115.0 \mathrm{mg} \mathrm{HA} / \mathrm{ccm}$ at 12 weeks. At both time points Group A densities were significantly higher than those of Group B, which were 290.1 \pm 61.0 $\mathrm{mg} \mathrm{HA} / \mathrm{ccm}$ at 4 weeks and $583.4 \pm 125.6 \mathrm{mg}$ $\mathrm{HA} / \mathrm{ccm}$ at 12 weeks $(p<0.05$, Fig. 6D). The regenerated bone volumes of Group A were also significantly higher than Group B: $120.9 \pm 22.2 \mathrm{~mm}^{3}$ versus $45.8 \pm 11.0 \mathrm{~mm}^{3}$ at 4 weeks and $205.0 \pm 32.8$ $\mathrm{mm}^{3}$ versus $103.0 \pm 20.1 \mathrm{~mm}^{3}$ at 12 weeks post-operation $(p<0.05$, Fig. $6 \mathrm{C})$.

\section{Histological assessment of bone regeneration and scaffold degradation}

Using H\&E staining we observed regeneration of numerous bone trabeculas at the bone defect areas in Group A at 4 weeks post-operation that closely connected the DCBS and autologous bone interface. The trabeculas grew into the DCBS, reaching the center part of the DCBS at the bone defect site and initiating scaffold degradation at the fibula side. No new bones were generated at the steel plate side during this observation period (Fig. 7A). At the same time, in Group B we observed some bone regeneration but the mineralization intensity and maturity of these new bones were lower than in Group A. Moreover, there was no evidence of regenerated bone entry into the DCBS center or fibula side, the scaffold was not degraded, and its pores were filled with fibroblasts (Fig. 7B).

At 12 weeks, post-operation the bone defects in Group A were filled by lamellar bones and the DCBS was fully degraded and replaced by recanalization of the medullary cavity and fat-like marrow tissue. Although new bone at the fibula showed good remodeling at 12 weeks in Group B and there was new bone generated on the surface of the central DCBS, there was no regeneration at the steel plate and some scaffold remained. We then used image analysis to determine the percent of the total defect area covered by regenerated bone. In Group A the average percentages of new bone in the total bone defect area at weeks 4 and 12 were $25.4 \% \pm 4.2 \%$ and $38.6 \% \pm$ $6.3 \%$, significantly higher than the $7.6 \% \pm 2.2 \%$ and $20.4 \% \pm 4.2 \%$ in Group B at the same time points $(p<0.05$, Fig. $7 \mathrm{C})$. We also detected significantly less DCBS as a percent of the total bone defect area in Group A compared to Group B: $15.1 \% \pm 3.7 \%$ in A versus $27.5 \% \pm 2.4 \%$ in $B$ at 4 weeks and $1.3 \% \pm 0.9 \%$ versus $19.7 \% \pm 4.2 \%$, at 12 weeks $(p<0.05$, Fig. $7 D)$. 
4 week
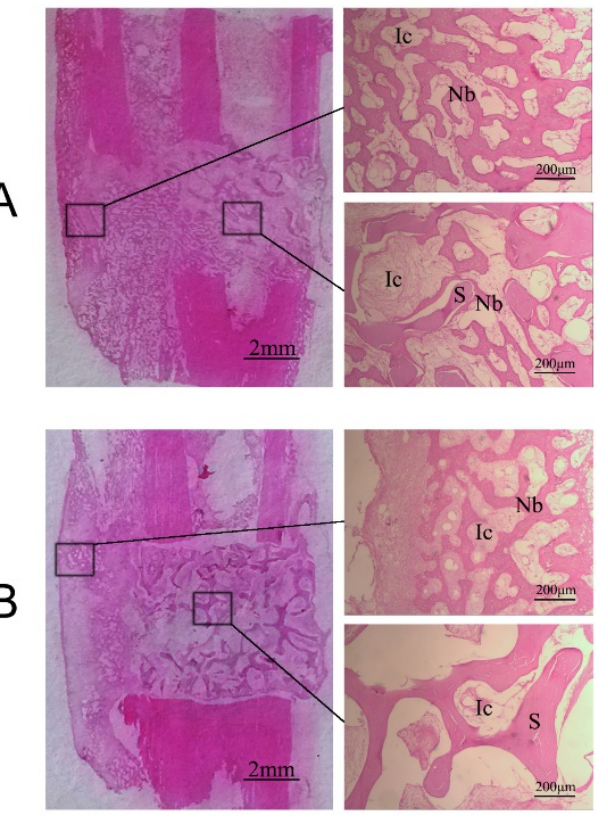

C

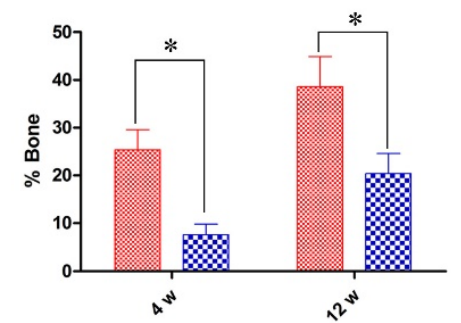

12 week
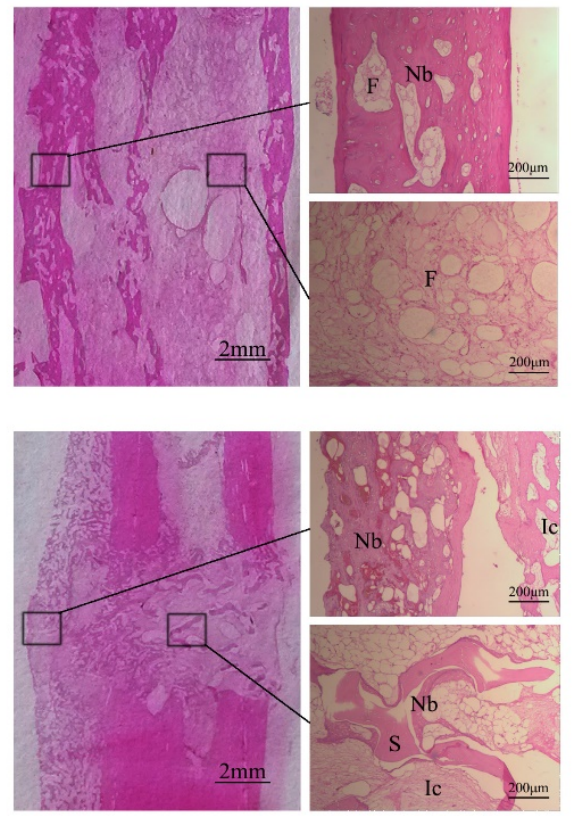

$\mathrm{D}$

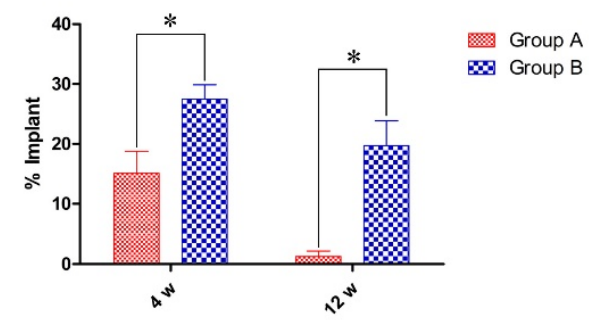

Figure 7. Histological images and quantitative assessment of bone regeneration and DCBS degradation. Central histological slices taken from the fibula side of the defects show improved bone regeneration at 4 and 12 weeks post-operation in A: Group A compared to B: Group B (40x). Image analysis quantification showed C: significantly higher percentages of regenerated bones and D: significantly lower percentages of residual DCBS in the total bone defect area in Group A compared to Group B. Ic: interstitial cell, Nb: new bone, S: scaffold, F: fat tissue. $n=5, *_{p}<0.05$.

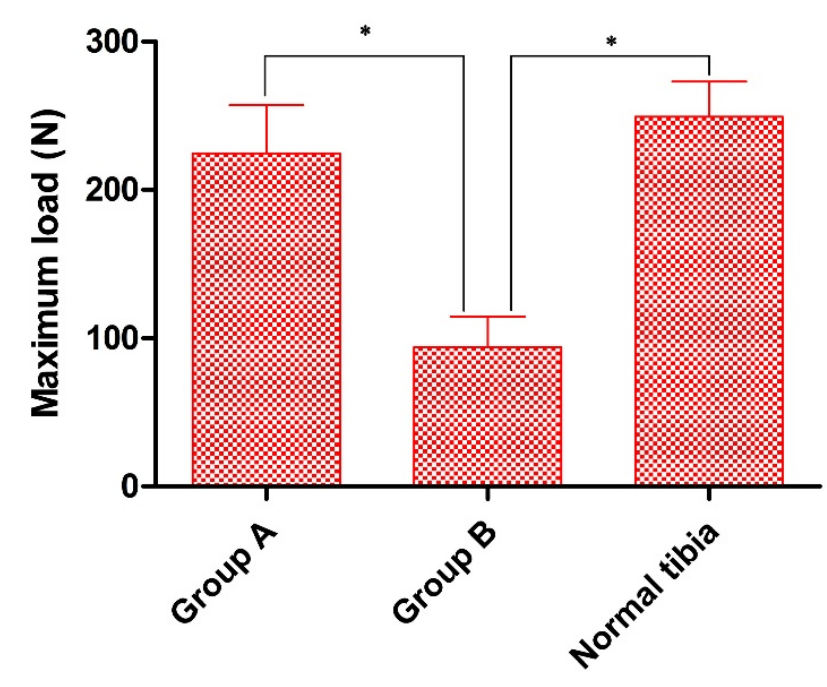

Figure 8. Biomechanical strength of bone defect sites. At $12 \mathrm{w}$ post-operation the maximum load strength of Group $A$ was significantly higher than that of Group B and similar to that of normal tibia. $n=5,{ }^{*} p<0.05$.

\section{Biomechanical testing of healed tibia}

Finally, at 12 weeks post-operation we performed a three-point bending test to determine new bone strength, with normal tibiae as controls. Although the maximum load for fracturing in Group A $(224.5 \pm 32.6 \mathrm{~N})$ was slightly below that of controls $(249.3 \pm 23.9 \mathrm{~N})$ the difference was not statistically significant. However, in Group. B the maximum endurable crack-strength was well below that of either Group A or the controls $(94.0 \pm 20.7 \mathrm{~N})$ (Fig. 8).

\section{Discussion}

Mechanical loading has been shown to alter the electrochemical environment of mesenchymal cells, stimulating bone callus formation and promoting bone joining and remodeling [14]. However, there is often an absence of mechanical loading in segmental bone defects because damage from severe trauma and strong fixation with instruments tend to cause local 
mechanical shielding. Patients may also have injuries in other organs that require extended immobilization during early recovery, or may avoid load-bearing exercise until late recovery fearing fixation breakage. Thus, methods restoring the mechanical loading environment in a controlled way during early recovery could greatly improve patient outcomes. The effect of cyclic loading on bone mineralization has been studied by Duty et al using a subcutaneous bone chamber seeded with a polymeric scaffold to study the effect of cyclic loading on mineralization [17], and Alireza et al studied the effect of cyclic loading on bone formation within a PLA/ $\beta$-TCP scaffold implanted in rat distal femoral epiphyses [9], but neither of these animal models is truly applicable to clinical situations. At the same time, fracture end mechanical stimulation with an invasive external fixator [15] and mechanical loading with a hydromechanic instrument [16] have been shown to restore the mechanical environment but whether such interventions are beneficial for bone regeneration in segmental bone defects remains unknown. In contrast to these previous studies, we employed a novel animal model of segmental bone defect and fixation using a tissue-engineered scaffold with plates and screws that more closely mimics clinical situations and applications.

In a previous study we designed a heel-strike like stress stimulator combining compressive and vibrative mechanical loading methods, and our results suggested this approach could improve outcomes for patients with non-union in the lower extremities [11]. Here, we employed a segmental bone defect model to investigate whether this mechanical loading method would also be beneficial for segmental bone defects after bone substitute grafting and its complications. We grafted DCBS, a commonly used bone substitute in clinical practices, into segmental tibial defects in rabbits and used our rap stress stimulator to apply heel-strike like mechanical loading to the tibial ends after surgery. Bone regeneration and DCBS scaffold degradation were tracked post-operation by X-ray examination, micro-CT scanning, histological staining, and a maximal load mechanical test and the results were analyzed quantitatively. Data from each of these approaches demonstrated that heel-strike like mechanical loading significantly accelerated bone regeneration and DCBS degradation compared to grafting alone without causing any complications. In both Group A and B the bone was subjected to static mechanical stimulation generated by muscle contraction and gravity despite immobilization by external plaster fixation. Static mechanical loading has also been shown to facilitate bone growth [18], but callus formation is more sensitive to dynamic, cyclic mechanical stimulation than static mechanical stimulation [19]. Thus, the DCBS scaffolds in Group A degraded more quickly than those of Group B and bone regeneration in Group A occurred at a faster rate. These results suggest that our heel-strike mechanical loading method can provide dynamic cyclic mechanical stimulation to restore the mechanical loading environment in segmental bone defects and facilitate bone regeneration and scaffold degradation.

When external mechanical loading should begin post-surgery remains under debate. Previous research has suggested that early application of mechanical stimulation can accelerate fracture healing [20], but premature mechanical loading may aggravate pain, fracture section swelling, wound exudation, and inflammation. Functional weight-bearing beginning the first day after bone graft surgery has been shown to reduce vascularization at bone defect sites by $66 \%$ and osteogenesis by $75 \%$ in rats after two weeks [21]. More recent studies, including those of Goodship and Kershaw, suggest the optimal time to initiate mechanical treatment is the first week after fracture [22],[23]. Interestingly, Ghias et al. reported that application of mechanical stimulation three days after operation hindered callus formation at bone defect areas in rats, whereas delaying treatment until two weeks post-operation facilitated the growth of new callus [9]. Our results are consistent with this report, as we found that initiating heel-strike mechanical loading stimulation one week post-operation caused no apparent damage during bone defect repair and significantly enhanced bone regeneration long-term.

The effect of mechanical loading on bone scaffolds might be influenced by various factors, including continuity of the bone defect ends. Destruction of the mechanical loading environment in a segmental bone defect is more serious than in simple bone fracture and grafting tissue-engineered bone substitutes can only partially restore continuity. In addition, the hardness and elasticity of the grafted bone substitute material can influence transmission of mechanical loading, while internal and external fixation materials may provide shielding that significantly absorbs mechanical loading [24]. In our study, we found that bone regeneration in Group A occurred at the fibula side opposite the steel plate during the early stages of recovery and regenerated bones gradually grew into the steel plate side over time. This was most likely due to eccentric fixation of the steel plates, which provided mechanical shielding that distributed loading in the DCBS scaffold in a ladder pattern. Bone defect areas closer to the steel plates absorbed less mechanical loading stimulation 
and produced less micro motion, whereas areas farther from the steel plates produced more micro motion [24].

Mechanical loading intensity and frequency are also critical factors, and while a range of intensity and frequency has been documented $[15,16,25]$ there remains no agreement on the optimal conditions. Although we found in this study that a 1/2 load rap mechanical of the rabbit weight $(15 \mathrm{~N})$ improved bone regeneration after DCBS grafting, we cannot conclude from our results that this load is optimal. Previous experiments have shown that mechanical frequencies higher than $0.2 \mathrm{~Hz}$ could induce physiological bone growth of rat tibia [26], while Warden et al have suggested low-frequency stimulated micro motion best facilitates callus growth and accelerates mineralization, with $1-5 \mathrm{~Hz}$ as the optimal frequency [27]. While our results are consistent with this, future research will be necessary to determine whether optimal intensity and frequency of mechanical loading should conform to psychological changes and gradually increase during fracture healing.

\section{Conclusions}

The heel-strike like mechanical loading method we present here can restore the loading environment in segmental bone defects and facilitate bone regeneration after bone substitute grafting. This method is noninvasive, convenient, safe, and highly efficient, and may thus be an important addition to improve outcomes when tissue-engineered bone substitutes are used in clinical settings.

\section{Acknowledgments}

This project was supported by the National Natural Science Foundation of China (Grant no. 81401783, 81371951) and the Youth Fund for Science and Technology (Grant no. 2016J05208, 13QNP048, 18FBQN2014006).

\section{Competing Interests}

The authors have declared that no competing interests exist.

\section{References}

[1] Dumic-Cule I, Pecina M, Jelic M, et al. Biological aspects of segmental bone defects management. Int Orthop. 2015; 39:1005-1011.

[2] Amini AR, Laurencin CT, Nukavarapu SP. Bone tissue engineering: recent advances and challenges. Crit Rev Biomed Eng. 2012; 40: 363-408.

[3] Liu Y, Ming L, Luo H, et al. Integration of a calcined bovine bone and BMSC-sheet 3D scaffold and the promotion of bone regeneration in large defects. Biomaterials. 2013; 34:9998-10006.

[4] Li H, Ji Q, Chen X, et al. Accelerated bony defect healing based on chitosan thermosensitive hydrogel scaffolds embedded with chitosan nanoparticles for the delivery of BMP2 plasmid DNA. J Biomed Mater Res A. 2017; 105:265-273.

[5] Vahabi S, Torshabi M, Esmaeil Nejad A. In vitro comparison of the efficacy of TGF- $\beta 1$ and PDGF-BB in combination with freeze-dried bone allografts for induction of osteogenic differentiation in MG-63 osteoblast-like cells. J Mater Sci Mater Med. 2016; 27:182.
[6] Fan $H$, Zeng $X$, Wang $X$, et al. Efficacy of prevascularization for segmental bone defect repair using $\beta$-tricalcium phosphate scaffold in rhesus monkey. Biomaterials. 2014; 35:7407-7415

[7] Boerckel JD, Kolambkar YM, Stevens HY, et al. Effects of in vivo mechanical loading on large bone defect regeneration. J Orthop Res. 2012; 30:1067-1075.

[8] Tanaka SM, Sun HB, Roeder RK, et al. Osteoblast responses one hour after load-induced fluid flow in a three-dimensional porous matrix. Calcif Tissue Int. 2005; 76:261-271.

[9] Roshan-Ghias A, Terrier A, Bourban PE, et al. In vivo cyclic loading as a potent stimulatory signal for bone formation inside tissue engineering scaffold. Eur Cell Mater. 2010; 19:41-49.

[10] Wei FY, Leung KS, Li G, et al. Low intensity pulsed ultrasound enhanced mesenchymal stem cell recruitment through stromal derived factor-1 signaling in fracture healing. PLoS One. 2014; 9:e106722.

[11] Yao JF, Shen JZ, Li DK, et al. Rap system of stress stimulation can promote bone union after lower tibial bone fracture: a clinical research. Int J Med Sci. 2012; 9:462-466.

[12] Gao J, Huang G, Liu G, et al. A biodegradable antibiotic-eluting PLGA nanofiber-loaded deproteinized bone for treatment of infected rabbit bone defects. J Biomater Appl. 2016; 31:241-249.

[13] Baumann AP, Aref MW, Turnbull TL, et al. Development of an in vivo rabbit ulnar loading model. Bone. 2015; 75:55-61.

[14] Chen JH, Liu C, You L, et al. Boning up on Wolff's Law: mechanical regulation of the cells that make and maintain bone. J Biomech. 2010;43: 108-118.

[15] Schwarz C, Wulsten D, Ellinghaus A, et al. Mechanical load modulates the stimulatory effect of BMP2 in a rat nonunion model. Tissue Eng Part A. 2013; 19: 247-254.

[16] Boerckel JD, Kolambkar YM, Stevens HY, et al. Effects of in vivo mechanical loading on large bone defect regeneration. J Orthop Res. 2014; 30: 1067-1075.

[17] Duty AO, Oest ME, Guldberg RE. Cyclic mechanical compression increases mineralization of cell-seeded polymer scaffolds in vivo. J Biomech Eng. 2007; 29:531-539.

[18] Robling AG, Duijvelaar KM, Geevers JV, et al. Modulation of appositional and longitudinal bone growth in the rat ulna by applied static and dynamic force. Bone. 2001; 29: 105-113.

[19] Altmann B, Löchner A, Swain M, et al. Differences in morphogenesis of 3D cultured primary human osteoblasts under static and microfluidic growth conditions. Biomaterials. 2014; 35: 3208-3219.

[20] Marsell R, Einhorn TA. The biology of fracture healing. Injury. 2011; 42: 551-555.

[21] Wallace AL, Draper ER, Strachan RK, et al. The vascular response to fracture micromovement. Clin Orthop Relat Res. 1994; 301: 281-290.

[22] Goodship AE, Kenwright J. The influence of induced micromovement upon the healing of experimental tibial fractures. J Bone Joint Surg Br. 1985; 67: 650-655.

[23] Kershaw CJ, Cunningham JL, Kenwright J. Tibial external fixation, weight bearing and fracture movement. Clin Orthop. 1993; 293: 26-28.

[24] Claes L. Biomechanical principles and mechanobiologic aspects of flexible and locked plating. J Orthop Trauma. 2011; 25: S4-S7.

[25] Boerckel JD, Uhrig BA, Willett NJ, et al. Mechanical regulation of vascular growth and tissue regeneration in vivo. Proc Natl Acad Sci USA. 2011; 108: E674-680.

[26] Turner CH, Forwood MR, Otter MW. Mechanotransduction in bone: do bone cells act as sensors of fluid flow? FASEB J. 1994; 8:875-878.

[27] Warden SJ, Turner $\mathrm{CH}$. Mechanotransduction in the cortical bone is most efficient at loading frequencies of 5-10 Hz. Bone. 2004; 34: 261-270. 